\title{
High risk human papillomavirus viral load and persistence among heterosexual HIV-negative and HIV-positive men
}

\author{
Mary K. Grabowski ${ }^{1}$, Ronald H Gray ${ }^{1,2}$, David Serwadda ${ }^{2,3}$, Godfrey Kigozi ${ }^{2}$, Patti E. \\ Gravitt $^{1,4}$, Fred Nalugoda ${ }^{2}$, Steven J. Reynolds ${ }^{1,2,5,8}$, Maria J. Wawer ${ }^{1,2}$, Stephen Watya ${ }^{6}$, \\ Thomas C. Quinn ${ }^{2,5,8}$, and Aaron A. R. Tobian ${ }^{2,7}$
}

${ }^{1}$ Department of Epidemiology, Johns Hopkins University, Bloomberg School of Public Health, Baltimore, Maryland ${ }^{2}$ Rakai Health Sciences Program, Entebbe, Uganda ${ }^{3}$ School of Public Health, Makerere University, Kampala, Uganda ${ }^{4}$ Perdana University Graduate School of Medicine, Serdang, Malaysia ${ }^{5}$ Division of Intramural Research, National Institute of Allergy and Infectious Diseases, National Institutes of Health, Bethesda, Maryland, USA ${ }^{6}$ Department of Urology, Makerere University, Kampala, Uganda ${ }^{7}$ Department of Pathology, School of Medicine, Johns Hopkins University, Baltimore, Maryland ${ }^{8}$ Department of Medicine, School of Medicine, Johns Hopkins University, Baltimore, Maryland

\section{Abstract}

Objectives-High-risk human papillomavirus (HR-HPV) viral load is associated with HR-HPV transmission and HR-HPV persistence in women. It is unknown whether HR-HPV viral load is associated with persistence in HIV-negative or HIV-positive men.

Methods-HR-HPV viral load and persistence were evaluated among 703 HIV-negative and 233 HIV-positive heterosexual men who participated in a male circumcision trial in Rakai, Uganda. Penile swabs were tested at baseline and 6,12 and 24 months for HR-HPV using the Roche HPV Linear Array, which provides a semi-quantitative measure of HPV shedding by hybridization band intensity (graded:1-4). Prevalence risk ratios (PRR) were used to estimate the association between HR-HPV viral load and persistent detection of HR-HPV.

Results-HR-HPV genotypes with high viral load (grade:3-4) at baseline were more likely to persist than HR-HPV genotypes with low viral load (grade:1-2) among HIV-negative men (month 6: adjPRR=1.83, 95\%CI:1.32-2.52; month 12: adjPRR=2.01, 95\%CI:1.42-3.11), and HIVpositive men (month 6: adjPRR=1.33, 95\%CI:1.06-1.67; month 12: adjPRR=1.73, 95\%CI:1.182.54). Long-term persistence of HR-HPV was more frequent among HIV-positive men compared

Corresponding Author: Aaron Tobian, MD, PhD, Department of Pathology, Johns Hopkins University, Carnegie 437, 600 N. Wolfe St., Baltimore, MD 21287, 443-287-0527, atobian1@jhmi.edu.

CONFLICT OF INTEREST STATEMENT

There are no other potential conflicts of interest relevant to this article.

AUTHOR CONTRIBUTIONS

All authors contributed to the study design, data collection, data analysis, writing and reviewing the paper.

Except for Thomas Quinn who is an NIH employee, the Corresponding Author has the right to grant on behalf of all authors and does grant on behalf of all authors, an exclusive licence on a worldwide basis to the BMJ Publishing Group Ltd to permit this article (if accepted) to be published in STI and any other BMJPGL products and sub-licences such use and exploit all subsidiary rights, as set out in our licence http://group.bmj.com/products/journals/instructions-for-authors/licence-forms. 
to HIV-negative men (month 24: adjPRR=2.27, 95\%CI: 1.47-3.51). Persistence of newly detected HR-HPV at the 6 and 12 month visits with high viral load were also more likely to persist to 24 months than HR-HPV with low viral load among HIV-negative men (adjPRR=1.67, 95\% CI 0.883.16).

Conclusions-HR-HPV genotypes with high viral load are more likely to persist among HIVnegative and HIV-positive men, though persistence was more common among HIV-positive men overall. The results may explain the association between high HR-HPV viral load and HR-HPV transmission.

\section{Keywords}

Human papillomavirus (HPV); human immunodeficiency virus (HIV); male circumcision; Uganda; penile cancer; sexually transmitted infections; viral shedding; viral load; linear array band intensity

\section{INTRODUCTION}

High risk human papillomavirus (HR-HPV) infection is a common sexually transmitted infection, and most sexually active individuals will acquire HR-HPV in their lifetime ${ }^{1,2}$. Persistent HR-HPV can cause oral, anal, penile, and cervical cancer, and greater than $85 \%$ of the disease burden is in developing countries ${ }^{2-4}$. Cervical cancer is the leading cause of cancer mortality in women in Eastern Africa ${ }^{4}$. Among women, numerous studies have demonstrated that HR-HPV DNA viral load is associated with both persistence ${ }^{5,6}$ and progression to cervical lesions, especially HR-HPV $16^{5,7}$.

Data on the natural history of HR-HPV infection in men is limited. HR-HPV infection may be associated with HIV acquisition in men ${ }^{8,}{ }^{9}$. In addition, HR-HPV persistence among heterosexual men is associated with HIV infection, smoking, increased number of lifetime sexual partners, absence of male circumcision, and younger age ${ }^{10-13}$. Higher viral load among men is associated with detection of HR-HPV at multiple penile sites ${ }^{14}$ and flat penile lesions ${ }^{15}$. It has also been shown that HR-HPV transmission to female partners is associated with higher male viral loads ${ }^{16}$. Since male HR-HPV infection is a key component of HPV transmission $^{2,17}$, it is important to understand the natural history of HR-HPV in both sexes. However, neither risk factors for increased HR-HPV viral load nor the association between HR-HPV viral load and persistent infection among heterosexual men have been evaluated. In this study, HR-HPV viral load and persistence were assessed among HIV-negative and HIV-positive heterosexual men who participated in a male circumcision trial in Rakai, Uganda.

\section{MATERIALS AND METHODS}

\section{Study design and participants}

HR-HPV was assessed among men aged 15-49 years during two trials of male circumcision for HIV and STI prevention in Rakai District, Uganda ${ }^{18,19}$. Men who had contraindications for surgery (e.g., anemia, active genital infection) were treated, and if their medical condition resolved, they were re-screened and enrolled into the trial. Those with anatomical 
abnormalities (e.g., hypospadias), other medical contraindications or indications for surgery (e.g., severe phimosis) were excluded. HIV-positive men with CD4 counts $<250$ cells $/ \mathrm{mm}^{3}$ or WHO stage 4 disease were excluded from the trials and referred for treatment.

Participants provided written informed consent prior to screening and at baseline. Men were randomly assigned to receive immediate circumcision (intervention) or circumcision delayed for 24 months (control). Male circumcision was performed following the baseline visit for those men in the treatment arm of the trial. Infectious disease testing (HPV, HIV, HSV-2, and syphilis), physical examinations, and interviews to ascertain sociodemographic characteristics and sexual risk behaviors were conducted at and at 6,12 and 24 months follow-up visits. Serum and swab samples were stored at $-80^{\circ} \mathrm{C}$. All subjects were offered free HIV counseling and testing, health education and condoms at each visits and those participants found to be HIV-positive were referred for free care to the Rakai Health Sciences Program.

Of 6396 men enrolled in the two trials, 1790 men with a total of 5478 samples were tested for HR-HPV. Males were randomly selected from the trial population, except for married men, who were oversampled to permit a parallel study of HPV transmission to their female partners ${ }^{13}$. There were 949 swabs (17.3\%) with no detectable cellular beta-globin or detectable HPV that were excluded in this analysis since the adequacy of the sample collection could not be ensured. HIV seroconverters $(n=97)$ and men with an indeterminate HIV result at last visit $(n=115)$ were excluded from this analysis since acute HIV infection is associated with a substantial increase of new HR-HPV infection ${ }^{20}$. Men with penile lesions and/or warts were included in our analyses. Of 1529 participants with 3961 observations, we restricted our analyses to only those men who provided swab samples at baseline and at least one follow-up visit (6,12 and/or 24 months follow-up). A total 3084 study visits from 936 participants (703 HIV-negative and 233 HIV-positive men) were analyzed for this study.

Our primary analysis focused on the association between HR-HPV DNA load and the persistence of HR-HPV infections detected among HIV-negative and HIV positive men at baseline to 6,12 , and 24 months. We also conducted a secondary analysis in which we analyzed the association between HR-HPV viral load and persistence among men with newly detected infections at either month 6 and 12 to month 24 .

The trials were approved by the Ugandan National Council for Science and Technology (UNCST, Kampala, Uganda), and by three institutional review boards: the Science and Ethics Committee of the Uganda Virus Research Institute (Entebbe, Uganda), the Johns Hopkins University Bloomberg School of Public Health IRB (Baltimore, MD, USA), and the Western Institutional Review Board (Olympia, WA, USA). The trials were overseen by independent Data Safety Monitoring Boards ${ }^{21}$, and were registered with ClinicalTrials.Gov numbers NCT00425984 and NCT00124878.

\section{HR-HPV Detection, Viral load quantification, and STI Testing}

Moistened Dacron swabs were rotated around the full circumference of the penis at the coronal sulcus and glans by trained male clinicians or nurses, and were stored in Digene specimen transport media at $-80^{\circ} \mathrm{C}$ until assay. HPV genotyping was performed using the Roche HPV Linear Array (Roche Diagnostics, Indianapolis, IN) ${ }^{22}$. HPV genotypes 16, 18 , 
$31,33,35,39,45,51,52,56,58,59,66$, and 68 were considered high risk genotypes (HRHPV). For each positive HR-HPV genotype, the band intensity was visually scored as 1-4, with intensity 4 representing the strongest LA hybridization. All laboratory technologists and evaluators of band intensity were blinded to trial arm and all demographic data.

It has been previously demonstrated in both men and women that the linear array hybridization signal is linearly correlated with log-transformed HPV viral load (Spearman's $\mathrm{r}=073)^{23}$, 24; a linear array band signal strength of 4 is approximately equivalent to a viral load of $\geq 2000$ copies $/ 5 \mu \mathrm{l}$. Band signal strength of 3 is approximately $200-2000$ copies $/ 5 \mu \mathrm{L}$. Thus, band signals of 3 and 4 represent $>200$ copies $/ 5 \mu \mathrm{L}$. The linear array results and band intensity were evaluated by two observers, independently, using a labelled acetate overlay that was provided by Roche, where the overlay indicated the position of the genotype probes on the test strip. Observer disagreement occurred rarely (1-4\% of results) and were resolved by re-evaluation by the initial observers ${ }^{23}$. Here, we estimated the proportion of linear array results with band intensities 3 and 4 (i.e. high viral load HR-HPV), relative to lower intensity bands 1-2 (i.e. low viral load HR-HPV), among men with detectable HR-HPV infections.

HSV-2 infection was determined by HSV-2 ELISA (Kalon Biological Ltd, Guilford, UK), as previously described ${ }^{18}$. HIV status was determined using two separate ELISAs and confirmed by HIV-1 Western Blot, as previously described ${ }^{19}$.

\section{Statistical Analysis}

Baseline and follow-up characteristics of ART-naïve HIV-positive and HIV-negative men evaluated for HR-HPV were tabulated ( $n=936$ ). Men were stratified by HIV and HR-HPV status, and differences were assessed using chi-squared tests with two-sided p-values. In all other analyses, the unit of observation was the HR-HPV genotype. Persistent detection of HR-HPV infection was defined as an initially HR-HPV infected man with continued detection of same genotype at the 6,12 , or 24 month follow-up visits. All analyses were stratified by HIV-status and were conducted independently for each follow-up visit.

High viral load (band intensities 3-4) or low viral load (band intensities 1-2) was assessed for each HR-HPV genotype at baseline and follow-up visits, irrespective of the number of HR-HPV infections per individual. High viral load at baseline was the primary exposure variable and persistent HR-HPV detection at month 6, 12, or 24 months was the primary outcome. In a secondary analysis of newly detected HR-HPV at months 6 and 12, the exposure variable was viral load at time of initial detection and the outcome was the persistent detection of the same genotype at month 24 . The sensitivity of the results to our exposure definitions was also assessed by comparing persistence with band intensity 4 vs. band intensities 1-3.

We considered time to event/clearance analyses; however we decided to not use this approach for several reasons. First, a substantial fraction of individuals in this study (163/936) had only one follow-up visit, and from this one follow-up visit alone we could not be confident that an individual had actually cleared a particular HPV genotype. Similarly, many individuals had follow-up through only one year, and without additional follow-up 
through 24 months, we were not confident in ascribing clearance outcomes. HPV detection is also a recurrent outcome in some study individuals (Supplemental Table 1), making time to event analyses of clearance and their interpretation more complicated. Lastly, it is unknown whether HPV-infected persons clear virus or virus is not detected because it remains in a latent, inactive state in the genital tract that is below the level of detection.

We analyzed the association between HR-HPV viral load at baseline and the demographic and behavioral characteristics of HR-HPV-infected men using prevalence risk ratios (PRR) at each of the three follow-up visits. PRRs and 95\% confidence intervals (CI) were visitspecific and were estimated using Poisson regression with generalized estimating equations (GEE) and robust variance to account for correlation between multiple HR-HPV within the same man. Since we were estimating the association between HR-HPV viral load and detection at each follow-up visit independently, no offset was incorporated into the regression models. All analyses were stratified by the HIV-status of the man from which the HR-HPV was isolated.

Covariates in adjusted analyses included male age at baseline, which was highly correlated with marital status, treatment arm (i.e. circumcised or not), detection of beta-globin at baseline (for primary analyses) or follow-up (for secondary analyses), and sex with nonmarital partners in the last year (time varying at 6,12, and 24 months). Covariates were considered potential confounders if they were associated with the exposure and or causally associated with persistent detection, the latter of which was determined from the previously published literature ${ }^{8-13,23}$. Duration between visits were not include in the adjusted model since individuals were considered to have missed a study visit if they did not attend the scheduled visit within two weeks of the scheduled date during the primary trial. Therefore, small differences in visit attendance within this window would be unlikely to affect study inference for prevalence of HR-HPV detection at 6,12 , or 24 month visits by viral load. We could not adjust for smoking status, since these data were not obtained in the primary clinical trial. All effect estimates presented in the main text are adjusted estimates unless otherwise noted. We also examined whether the association of viral load was modified by infection with multiple HR-HPV at baseline or follow-up, circumcision status, and HR-HPV genotype. Interaction terms were used to test for effect modification by multiple HR-HPV infections, circumcision, and HR-HPV genotype.

Statistical analyses were conducted in STATA Version 11.0 (STATA Corp LP, College Station, Texas) and R version 2.14 .

\section{RESULTS}

Among 703 HIV-negative and 233 ART-naïve HIV-positive men evaluated for HR-HPV at baseline (Supplemental Table 2), at least one HR-HPV was identified in 264 HIV-negative men (37.6\%, 403 genotypes total) and 164 HIV-positive men (70.4\%, 399 genotypes total). Only one (55.8\%) or two HR-HPV (20.8\%) were detected in most men, however up to 9 unique HR-HPV genotypes were identified in a single study participant. The most frequently detected HR-HPV genotypes were HPV-16 (11.0\%) and HPV-51 (11.5\%) (Supplemental Table 3). 
Of the HR-HPV genotypes detected at baseline, $45.9 \%$ and $50.4 \%$ had a band intensity of 34 in HIV-positive and HIV-negative men, respectively (unadjusted PRR $=0.91,95 \% \mathrm{CI}$ : 0.74-1.16). Table 1 shows demographic and behavioral factors associated with high HRHPV viral load at baseline. Among HIV-negative men, infections with high viral load were detected more frequently in individuals who were younger and unmarried. There were no significant associations between a high HR-HPV viral load and baseline demographic or behavioral factors among HIV-positive men.

Persistent detection of HR-HPV at follow-up was significantly associated with a higher viral load at baseline (Table 2). Among HIV-negative men, HR-HPV infections with high viral loads were 1.83 times more likely to be detected at month 6 (95\% CI: $1.32-2.52)$ and 2.01 times more likely to be detected at month 12 (95\% CI: 1.42-3.11) than infections with low viral load at baseline. By month 24, differences between high and low viral load infections among HIV-negative men were no longer statistically significant; though, the prevalence of HR-HPV with high viral load at baseline was still greater than HR-HPV with low viral load (15.2\% vs. $8.8 \%$, respectively, adjPRR=1.58, 95\%CI:0.89-2.80).

Higher viral load at baseline was also significantly associated with persistent HR-HPV detection among HIV-positive men up to 12 months (Table 2). Prevalence of HR-HPVs with a high viral load at baseline were 1.33 times greater at 6 months (95\% CI: 1.06-1.67) and 1.73 times greater at 12 months (95\% CI: 1.18-2.54) than those HR-HPVs with low viral load. HR-HPV infections with low viral loads were detected more frequently at all followup visits in HIV-positive compared to HIV-negative men (6 months: adjPRR=1.86, 95\%CI: 1.15-3.03; 12 months: adjPRR=1.45, 95\%CI: 0.80-2.64; 24 months: adjPRR=2.72, 95\%CI: $1.42-5.20)$

Persistent detection of baseline HR-HPV at 24 months was more frequent among HIVpositive than HIV-negative men (Figure 1). At two years, detection of HR-HPV was 2.27 times greater among HIV-positive after controlling for HR-HPV viral load at baseline, circumcision status, detection of beta-globin at baseline, non-marital sexual partners in the last 12 months, and age (95\%CI:1.47-3.51). In our interaction analyses, the association between viral load and persistent HR-HPV detection during follow-up among HIV-negative or HIV-positive men did not differ by circumcision status, HR-HPV genotype, or infection with multiple HR-HPV at baseline or follow-up (data not shown), though we may have had limited power to detect differences among these subgroups.

Among HIV-negative men with HR-HPV at baseline and complete follow-up ( $\mathrm{n}=139 \mathrm{men}$ ), HR-HPV with high viral load at baseline were 3.76 (95\%CI: 0.68-20.8) times more likely to be persistently detected at all follow-up visits (6, 12, and 24 months). Similarly, the persistent detection of HR-HPV with high viral load at baseline was 1.79 (95\%CI: 1.003.23) times greater than for HR-HPV with low viral load at baseline among HIV-positive men ( $\mathrm{n}=86$ men).

Persistence of newly detected infections in month 6 and 12 was also analyzed and associated with viral load at initial detection (Table 3). There were 163 new HR-HPVs at month 6 and month 12 in 141 HIV-positive men, and 226 new infections in 87 HIV-negative men at the 
same visits. Associations between viral load and persistent detection in follow-up followed similar trends to that observed among baseline HR-HPV infections. Persistent detection of newly detected HR-HPV with high viral load more frequent than detection of new infections with low viral load among HIV-negative men; however, these results were not statistically significant (adjPRR=1.67, 95\% CI: 0.88-3.16). High viral load viruses detected at 6 and 12 months were not more likely to persist than low viral load viruses among HIV-positive men $(\operatorname{adjPRR}=1.10,95 \%$ CI: $0.67-1.80)$.

\section{DISCUSSION}

The natural history of HR-HPV is poorly characterized among HIV-negative and HIVpositive men, especially in resource limited settings where the burden of HR-HPV associated diseases is greatest ${ }^{4}$. The highest rates of penile cancers are found in sub-Saharan Africa, where both HR-HPV and HIV prevalence exceed that of all regions globally 2,4 . Rates of cervical cancer in sub- Saharan Africa are also highest worldwide ${ }^{4}$, and are almost universally associated with HR-HPV. The HR-HPV infections that ultimately result in cervical cancer are regularly acquired from HR-HPV infected men ${ }^{2}, 16$.

Here, we examined the association between high viral load and persistence of HR-HPV over a two year period among heterosexual men in Rakai District, Uganda. Among HR-HPV identified at baseline and newly detected HR-HPV at follow-up, we found that high viral load was associated with 6 and 12 but not 24 month HR-HPV persistence among HIVnegative and HIV-positive men. Persistent HR-HPV infections were more common among HIV-positive than HIV-negative men, and viruses with low viral load were detected at higher levels among HIV-positive men at all follow-up study visits.

Most HR-HPV infections detected at the baseline visit were no longer detected after one year, however persistence of remaining infections was strongly and significantly associated with viral load up to 12 months. Though differences in prevalence at 24 months were not statistically significant, persistent detection of HR-HPV with high viral load at baseline was more frequent among HIV-positive and HIV-negative men after two years. Our findings are similar to reports among women in which both higher viral load at first detection and increasing viral load across study visits have been associated with long term persistence of HR-HPV and incidence of cervical lesions ${ }^{6,25,26}$. High-HR HPV viral load in men may be associated with transmission of HR-HPV to their female partners ${ }^{16}$, and may be due in part, to the longer duration of high viral load HR-HPV infections among males.

We also conducted a secondary analysis among men in whom HR-HPV was newly detected at 6 or 12 month visits. Such newly detected HR-HPV may be a combination of incident infection and reactivation of low-level persistent HR-HPV infection ${ }^{27}$. While prevalent HRHPV infections identified at baseline likely over represent persistent infections ${ }^{5,6}$, we observed very similar patterns in HR-HPV persistence between prevalent infections at baseline and new detections identified during follow-up suggesting that bias in the baseline results may be limited if most newly detected viruses in fact reflect incident infection. 
Some studies have found that HR-HPV 16 viral load is more strongly associated with persistent viral detection in women than viral load of other genotypes $5,7,28$, but we observed no significant differences in persistence between genotypes in this study, though power was limited. Our results also suggested that younger and unmarried men were more likely to have high viral load among HIV-negative men, possibly because limited prior exposure is associated with a less robust immunologic response to HR-HPV acquired for the first time.

HR-HPV persistence was more common among HIV-positive men and was associated with increased viral load. While viral load was significantly associated with persistent detection of HR-HPV initially detected at baseline in HIV-positive men, the effect estimates were lower than those observed among HIV-negative men. Moreover, we found no significant differences in HR-HPV persistence by viral load among newly detected HR-HPVs in HIVpositive men possibly because of reduced cellular immune responses needed for viral clearance and in the case of latent HPV infection, control of viral replication ${ }^{29}$. Clearance of HR-HPV is increased with circumcision among HIV-negative, but not among HIV-positive men $^{13}$. However, we found that the association between circumcision and HR-HPV persistence among HIV-negative men was independent of viral load. While a previous study showed that co-infection with multiple HPV modified the association between viral load and persistence among women ${ }^{6}$, we did not observe any significant differences between men with single or multiple infections.

There are inherent difficulties in the study interpretation of HPV epidemiology, particularly when the interval between sample collection is long. We may have misclassified persistent but low-level HR-HPV that were undetectable as cleared infection, and HR-HPV that were rapidly cleared and re-acquired during intervals as persistent infections. We also may have misclassified persistent infection as new events if we did not detect HR-HPV infection at the previous study visit. Newly detected virus may also reflect reactivation of latent HR-HPV infection after a loss of local immune response to viral infection, which is likely more common among those with increasing age and HIV co-infection ${ }^{27,29}$. Specifically, increased reactivation of latent HR-HPV in the presence of HIV co-infection could explain the lack of association between high viral load and persistent detection for newly detected virus in HIV-positive men observed here.

There were other limitations to this study. We did not have samples for all men at each follow-up visit, and we have previously shown detection of beta-globin is significantly lower among uncircumcised men suggesting difficulty obtaining cellular material following circumcision $^{18}$. Men with penile lesions and/or warts were not excluded from our analyses and this may have affected HPV viral load. In women, HPV persistence and progression to cervical lesions is associated with lower CD4 cell count ${ }^{30}$. However, only men with a CD4 cell count $>250$ cells $/ \mathrm{mm} 3$ were included in the original trials, limiting any conclusions on the role of CD4 cell count and persistence in this study. We use a dichotomous measure of HR-HPV viral load, though we assessed the sensitivity of the findings to this exposure definition and found very similar results when only those viruses with the highest band intensity were classified as high viral load. Our results were also robust to adjustment for behavioral and clinical variables that may have confounded the association between viral 
load and persistent detection in follow-up; however, we were not able to adjust for smoking which may have confounded our results to some extent.

In conclusion, we find that HR-HPV viral load is associated with persistent detection of HRHPV infections in HIV-negative and HIV-positive men. Our finding highlight the role of viral load in the natural history of HR-HPV in men, and may explain in part increased levels of HR-HPV detection and persistence among individuals co-infected with HIV.

\section{Acknowledgments}

We are most grateful to the study participants and the Rakai Community Advisory Board whose commitment and cooperation made this study possible.

\section{FINANCIAL SUPPORT}

The trials were funded by the National Institutes of Health (\#UO1AI51171) and the Bill and Melinda Gates Foundation (\#22006.02). National Institute of Allergy and Infectious Diseases (NIAID), NIH grants U01AI-068613, 3U01-AI075115-03S1, 1K23AI093152-01A1, R01AI087409-01A1 and the NIAID Intramural Program provided laboratory support. A.A.R.T. was supported by the NIH 1K23AI093152-01A1 and Doris Duke Charitable Foundation Clinician Scientist Development Award(\#22006.02). The Fogarty International Center (\#5D43TW001508 and \#2D43TW000010-19-AITRP) contributed to training.

Dr. Gravitt received research funding from Roche Molecular Diagnostics who manufacture the HPV genotyping test used in this study.

\section{References}

1. Tobian AA, Gray RH. Male foreskin and oncogenic human papillomavirus infection in men and their female partners. Future Microbiol. 2011; 6(7):739-745. [PubMed: 21797689]

2. Partridge JM, Koutsky LA. Genital human papillomavirus infection in men. Lancet Infect Dis. 2006; 6(1):21-23. [PubMed: 16377531]

3. Munoz N, Bosch FX, de Sanjose S, et al. Epidemiologic classification of human papillomavirus types associated with cervical cancer. The New England Journal of Medicine. 2003; 348(6):518527. [PubMed: 12571259]

4. Ferlay J, Shin HR, Bray F, et al. Estimates of worldwide burden of cancer in 2008: GLOBOCAN 2008. Int J Cancer. 2010; 127:2893-2917. [PubMed: 21351269]

5. Gravitt PE, Kovacic MB, Herrero R, et al. High load for most high risk human papillomavirus genotypes is associated with prevalent cervical cancer precursors but only HPV16 load predicts the development of incident disease. Int J Cancer. 2007; 121(12):2787-2793. [PubMed: 17722112]

6. Xi LF, Hughes JP, Edelstein ZR, et al. Human Papillomavirus (HPV) type 16 and type 18 DNA Loads at Baseline and Persistence of Type-Specific Infection during a 2-year follow-up. J Infect Dis. 2009; 200(11):1789-1797. [PubMed: 19848609]

7. Dalstein V, Riethmuller D, Pretet JL, et al. Persistence and load of high-risk HPV are predictors for development of high-grade cervical lesions: a longitudinal French cohort study. Int J Cancer. 2003; 106(3):396-403. [PubMed: 12845680]

8. Tobian AA, Graowski MK, Kigozi G, et al. Human papilloma virus clearance among males is associated with HIV acquisition and increased dendritic cell density in the foreskin. J Infect Dis. 2013; 207(11):1713-1722. [PubMed: 23345339]

9. Smith JS, Moses S, Hudgens MG, et al. Increased risk of HIV acquisition among Kenyan men with human papillomavirus infection. J Infect Dis. 2010; 201(11):1677-1685. [PubMed: 20415595]

10. Backes DM, Bleeker MC, Meijer CJ, et al. Male circumcision is associated with a lower prevalence of human papillomavirus-associated penile lesions among Kenyan men. Int J Cancer. 2012; 130(8):1888-1897. [PubMed: 21618520] 
11. Giuliano AR, Lu B, Nielson CM, et al. Age-specific prevalence, incidence, and duration of human papillomavirus infections in a cohort of 290 US men. J Infect Dis. 2008; 198(6):827-835. [PubMed: 18657037]

12. Giuliano AR, Lee JH, Fulp W, et al. Incidence and clearance of genital human papillomavirus infection in men (HIM): a cohort study. Lancet. 2011; 377(9769):932-940. [PubMed: 21367446]

13. Tobian AA, Kigozi G, Gravitt PE, et al. Human papillomavirus incidence and clearance among HIV-positive and HIV-negative men in Rakai, Uganda. AIDS. 2012; 26(12):1555-1565. [PubMed: 22441255]

14. Flores R, Lu B, Nielson C, et al. Correlates of human papillomavirus viral load with infection site in asymptomatic men. Cancer Epidemiol Biomarkers Prev. 2008; 17(12):3573-3576. [PubMed: 19064573]

15. Bleeker MC, Hogewoning CJ, Voorhorst FJ, et al. HPV-associated flat penile lesions in men of a non-STD hospital population: less frequent and smaller in size than in male sexual partners of women with CIN. Int J Cancer. 2005; 113(1):36-41. [PubMed: 15386360]

16. Bleeker MC, Berkhof J, Hogewoning CJ, et al. HPV type concordance in sexual couples determines the effect of condoms on regression of flat penile lesions. Br J Cancer. 2005; 92(8): 1388-1392. [PubMed: 15812547]

17. Widdice L, Ma Y, Jonte J, et al. Concordance and Transmission of Human Papillomavirus Within Heterosexual Couples Observed Over Short Intervals. J Infect Dis. 2013

18. Tobian AA, Serwadda D, Quinn TC, et al. Male circumcision for the prevention of HSV-2 and HPV infections and syphilis. N Engl J Med. 2009; 360(13):1298-1309. [PubMed: 19321868]

19. Gray RH, Kigozi G, Serwadda D, et al. Male circumcision for HIV prevention in men in Rakai, Uganda: a randomised trial. Lancet. 2007; 369(9562):657-666. [PubMed: 17321311]

20. Nowak RG, Gravitt PE, Morrison CS, et al. Increases in human papillomavirus detection during early HIV infection among women in Zimbabwe. J Infect Dis. 2011; 203(8):1182-1191. [PubMed: 21451006]

21. Gray RH, Kigozi G, Serwadda D, et al. Male circumcision for HIV prevention in men in Rakai, Uganda: a randomised trial. Lancet. 2007; 369(9562):657-666. [PubMed: 17321311]

22. Gravitt PE, Peyton CL, Apple RJ, et al. Genotyping of 27 human papillomavirus types by using L1 consensus PCR products by a single-hybridization, reverse line blot detection method. Journal of Clinical Microbiology. 1998; 36(10):3020-3027. [PubMed: 9738060]

23. Wilson L, Gravitt P, Tobian A, et al. Male circumcision reduces penile high risk human papillomavirus (HPV) viral load in a randomized clinical trial in Rakai, Uganda. Sex Transm Infect. 2013; 89(3):262-266. [PubMed: 23112341]

24. Wentzensen N, Gravitt PE, Long R, et al. Human papillomavirus load measured by linear array correlates with quantitative PCR in cervical cytology specimens. J Clin Microbiol. 2012; 50(5): 1564-1570. [PubMed: 22337992]

25. Marks M, Gravitt PE, Utaipat U, et al. Kinetics of DNA load predict HPV 16 viral clearance. J Clin Virol. 2011; 51(1):44-49. [PubMed: 21388867]

26. Monnier-Benoit S, Dalstein V, Riethmuller D, et al. Dynamics of HPV16 DNA load reflect the natural history of cervical HPV-associated lesions. J Clin Virol. 2006; 35(3):270-277. [PubMed: 16214397]

27. Gravitt PE, Rositch AF, Silver MI, et al. A cohort effect of the sexual revolution may be masking an increase in human papillomavirus detection at menopause in the United States. J Infect Dis. 2013; 207(2):272-280. [PubMed: 23242540]

28. Xi LF, Hughes JP, Castle PE, et al. Viral load in the natural history of human papillomavirus type 16 infection: a nested case-control study. J Infect Dis. 2011; 203(10):1425-1433. [PubMed: 21415020]

29. Stanley M. Immune responses to human papillomavirus. Vaccine. 2006; 24(Suppl 1):S16-S22. [PubMed: 16219398]

30. Strickler HD, Burk RD, Fazzari M, et al. Natural history and possible reactivation of human papillomavirus in human immunodeficiency virus-positive women. J Natl Cancer Inst. 2005; 97(8):577-586. [PubMed: 15840880] 


\section{Key Messages}

- This population based study of heterosexual men in rural Sub-Saharan Africa demonstrates that higher HR-HPV viral load is associated with persistent detection of HR-HPV in HIV-positive and HIV-negative men.

- Persistent detection of HR-HPV is more common in HIV-positive than HIVnegative men irrespective of HR-HPV viral load.

- HR-HPV viral load is higher in younger, unmarried HIV-negative men.

- A higher HR HPV viral load in men may be associated with transmission of HR-HPV to their female partners, and may be due in part, to the longer duration of these high viral load HR-HPV infections among males. 


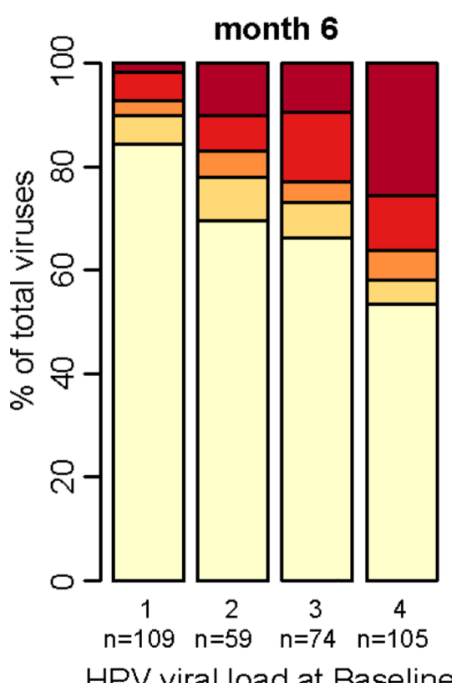

HIV-negative
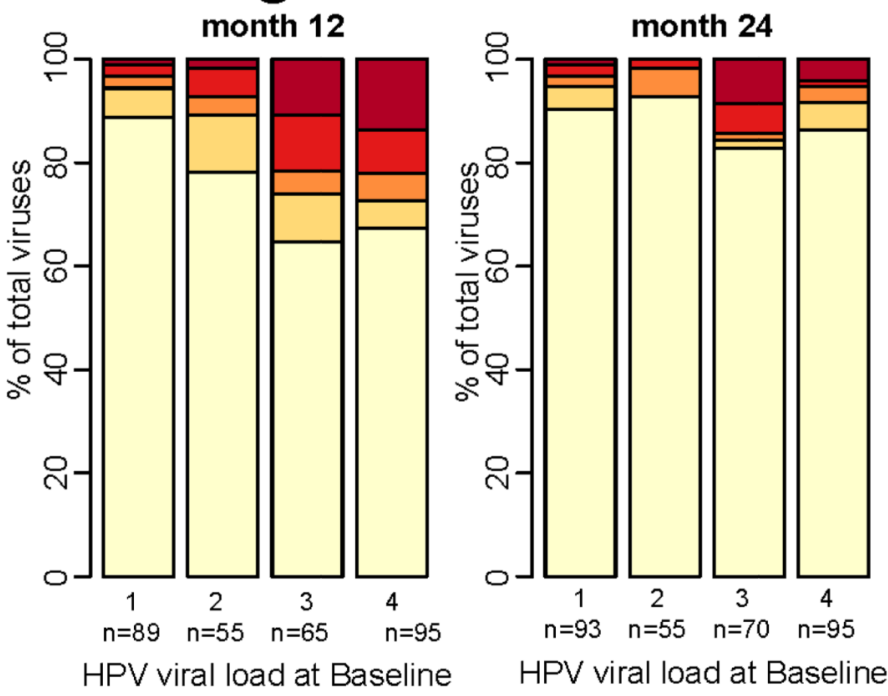

HPV viral load at follow-up

$\square$ Level 4

$\square$ Level 3

$\square$ Level 2

$\square$ Level 1

$\square$ Undetectable

\section{HIV-positive}
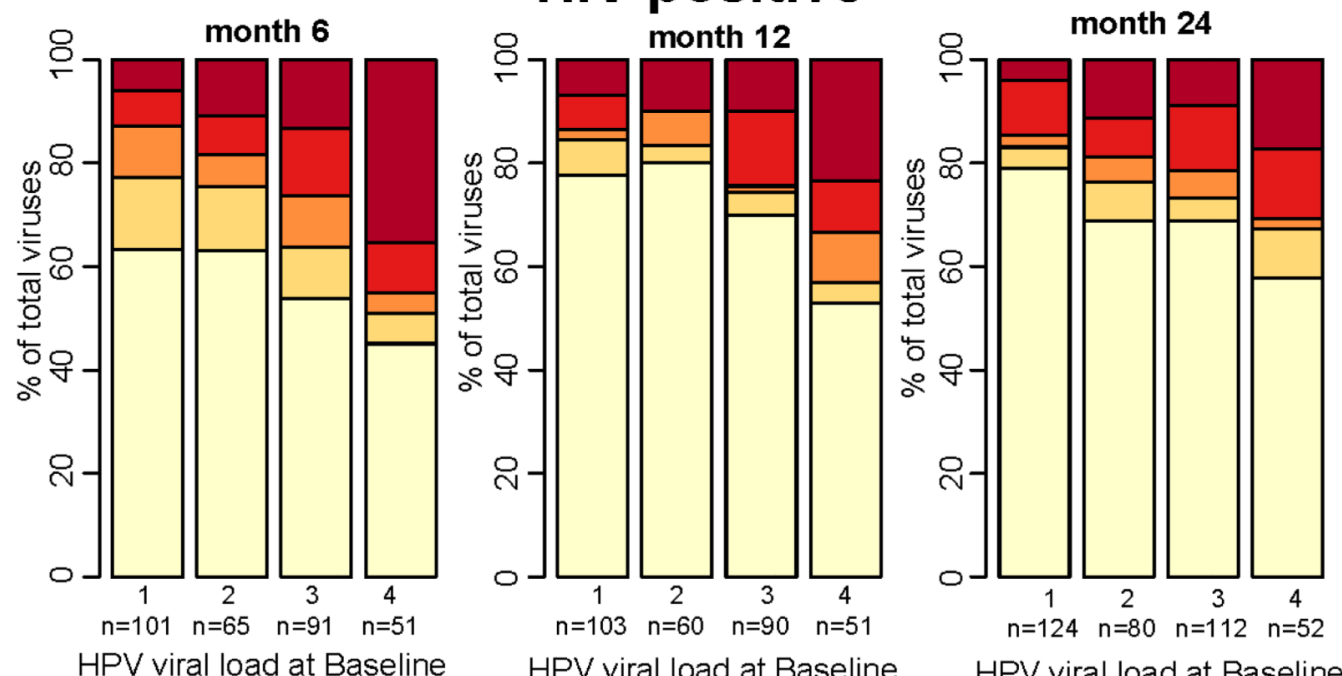

Figure 1.

Viral load of HR-HPV detected at baseline at 6, 12, and 24 months follow-up visits by baseline HR-HPV viral load and HIV status. 


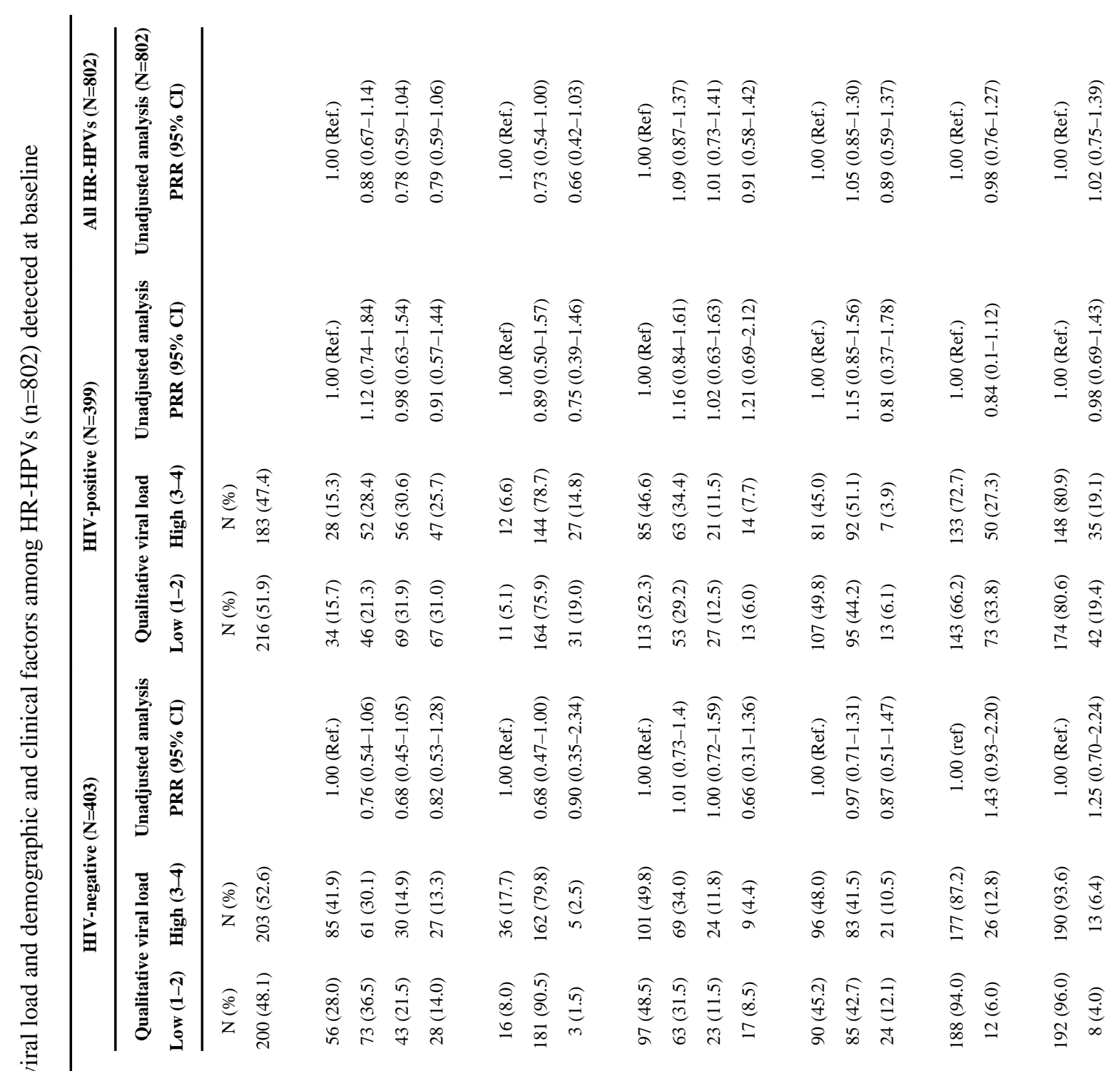

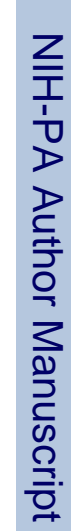
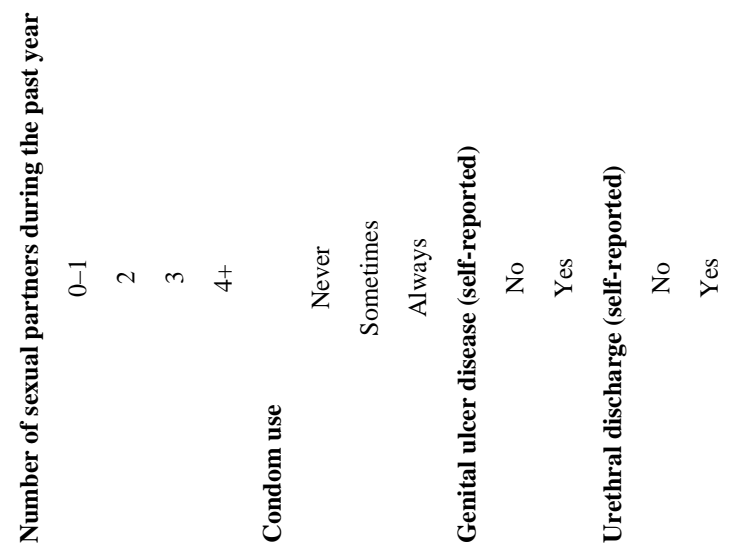


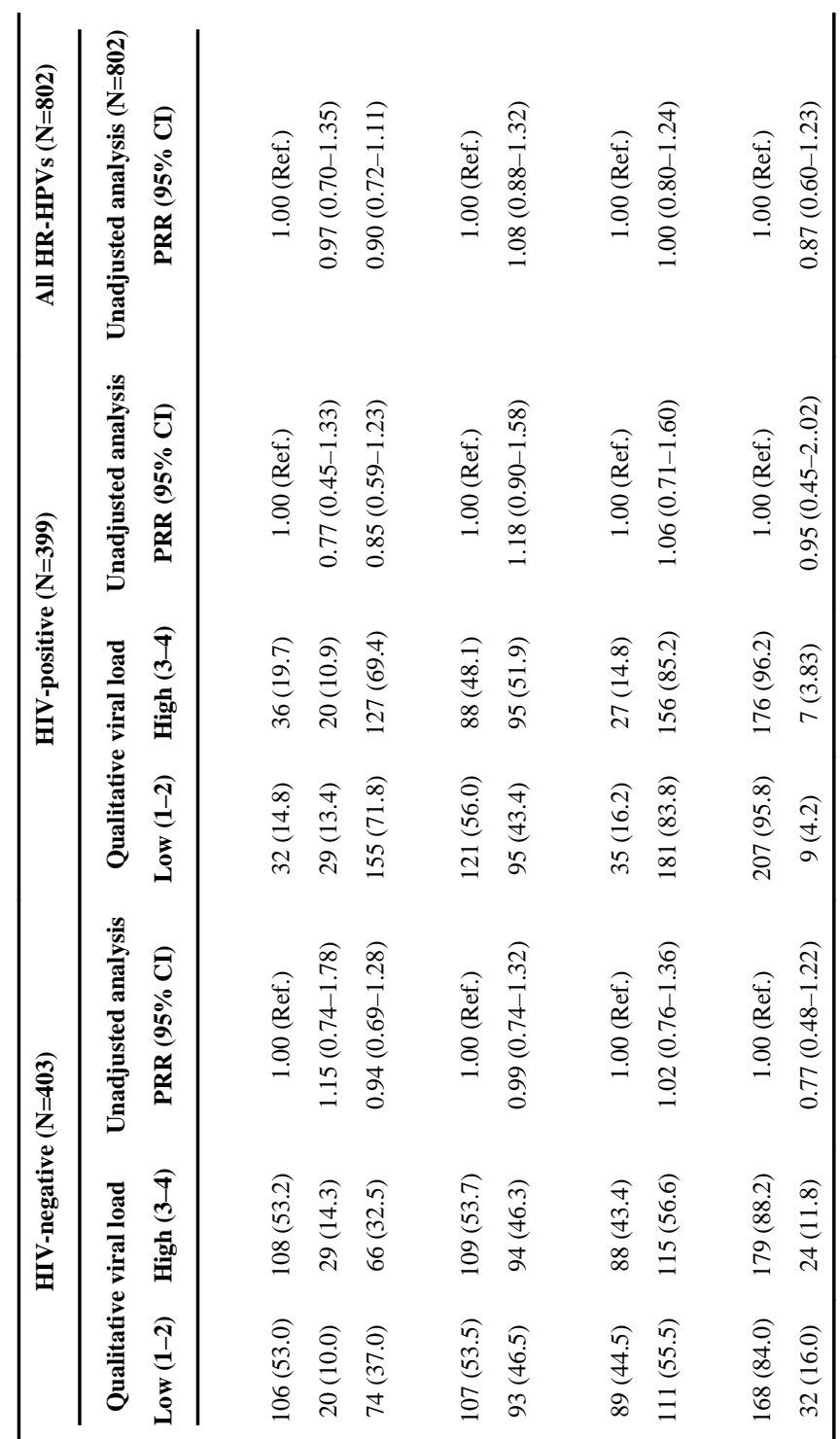

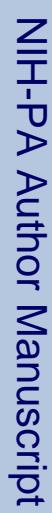

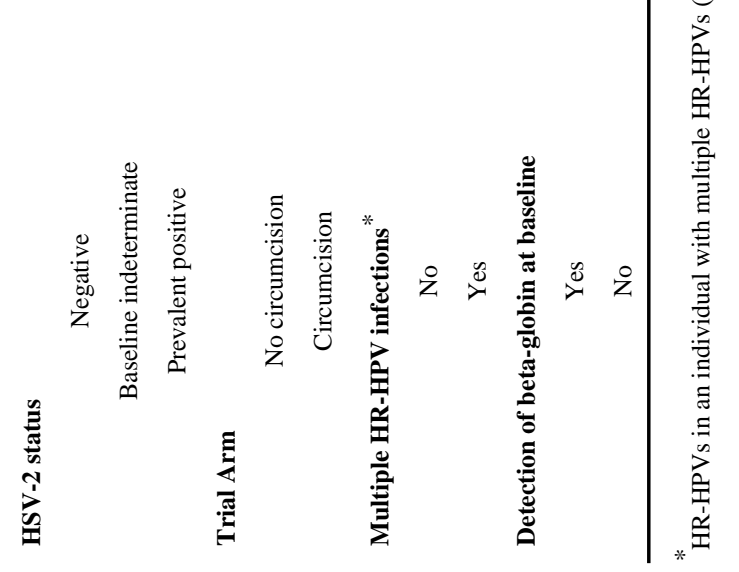




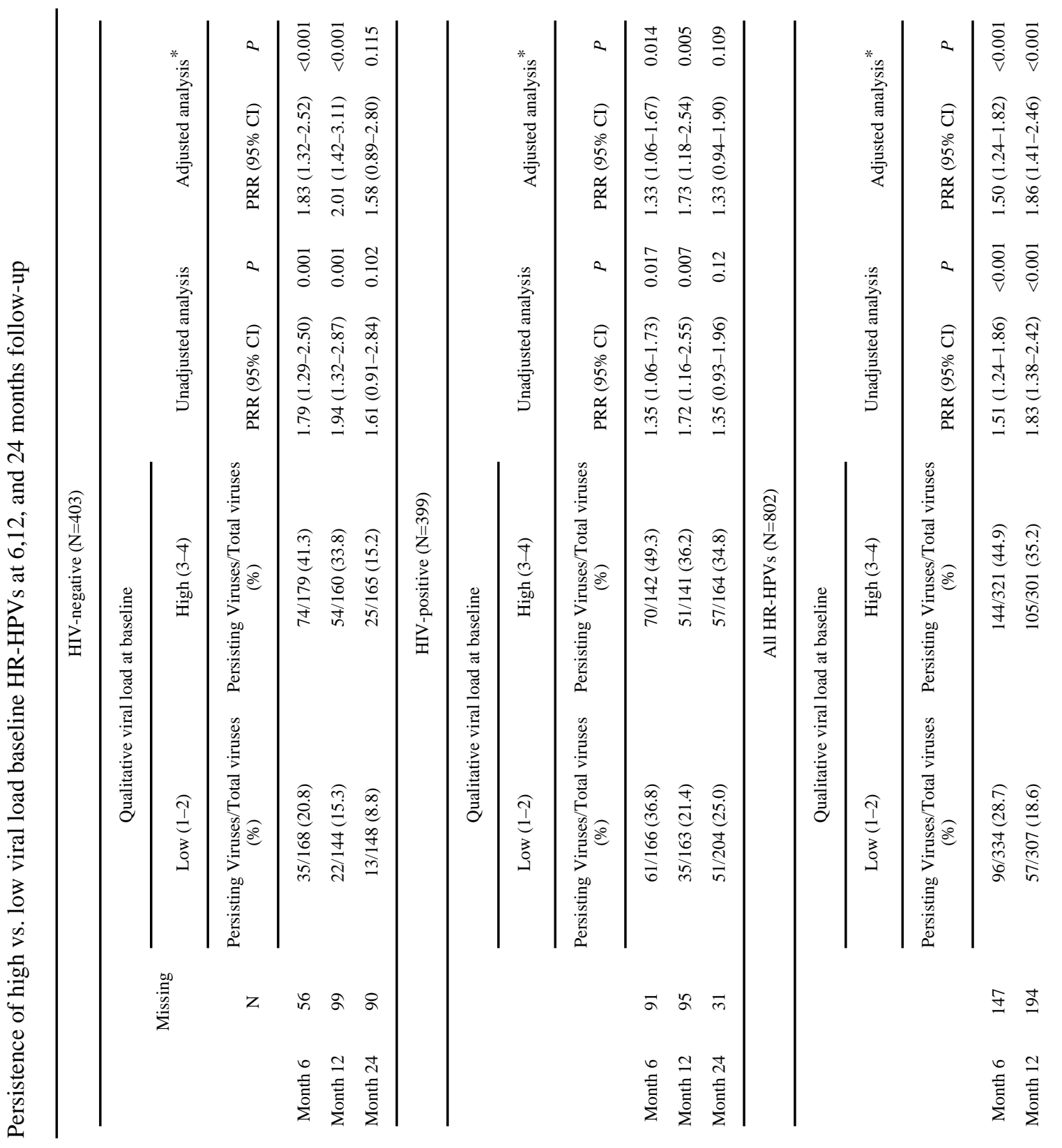




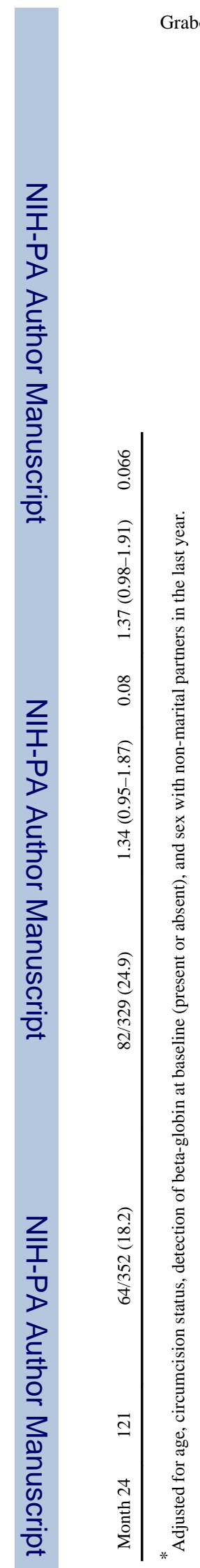

Page 16

Sex Transm Infect. Author manuscript; available in PMC 2014 June 01. 

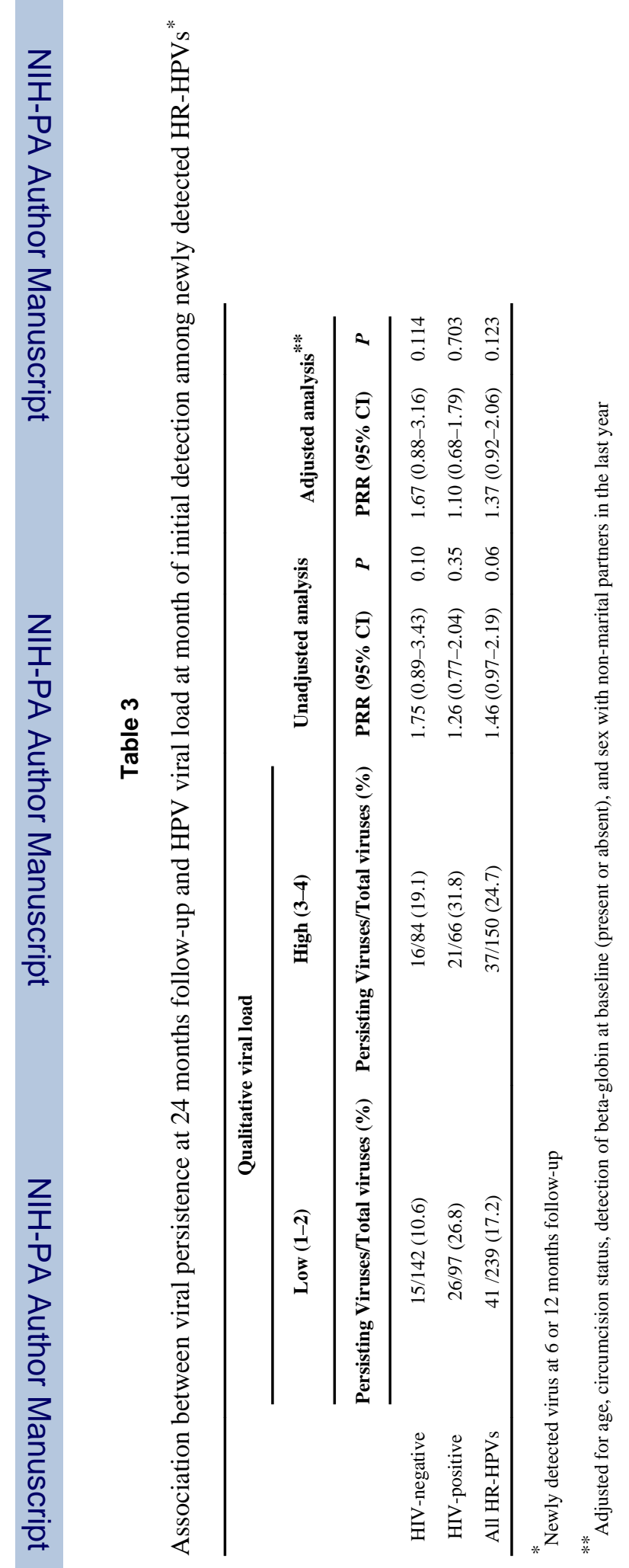

Sex Transm Infect. Author manuscript; available in PMC 2014 June 01. 